fall into one of four classes, according to the sequence of the quantities involved.

The geometric interpretation of a formula has been explained wherever possible, when used for the expression of an electromagnetic quantity, as mutual induction, magnetic potential, vector potential, and so forth; and the advantage of the Stokes function in analytic simplicity has been emphasized over the ordinary potential function.

F. N. CoLe, Secretary.

\title{
ON A SPECIAL ALGEBRAIC CURVE HAVING A NET OF MINIMUM ADJOINT CURVES.
}

BY PROFESSOR VIRGIL SNYDER.

(Read before the American Mathematical Society, September 5, 1907.)

Is researches on plane curves a very fruitful configuration has been employed by Küpper* in obtaining particular curves having double points in abnormal position. His method consists in using as basis points part of the intersections of two curves which satisfy certain prescribed conditions; pencils and nets are then constructed having these basis points as nodes. The question naturally arises whether the same procedure can be employed in other cases, making use of all the constants in the system. In the following note it will be shown that in such cases the special series which are obtained cannot be employed to reduce the order of the curve. Incidentally, an illustration is furnished of configurations having a $g_{2(n-1)}^{2}$ (a linear series with two degrees of freedom and of order $2(n-1))$, although the curve cannot be reduced to order $2(n-1)$.

1. Through $n-1$ points on a straight line pass two general curves of order $n, c_{n}, c_{n}^{\prime}$. These curves intersect in $n^{2}-n+1$ residual points through which can be passed $\infty^{2}$ curves of order $n$. Any two points $P, Q$ of the plane will determine two pencils $c_{n}+\lambda c_{n}^{\prime}=0, \phi_{n}+\lambda \phi_{n}^{\prime}=0$ contained in this net, which can so be made projective that the locus of the variable intersections is the curve $c_{2 n}$ of order $2 n$

$$
c_{n} \phi_{n}^{\prime}-c_{n}^{\prime} \phi_{n}=0 \text {. }
$$

* C. Küpper : "Ueber das Vorkommen von linearen Schaaren . . . ," Sitzungsberichte der Böhmischen Gesellschaft, Prag, 1892, pp. 264-272. 
The $n^{2}-n+1$ basis points are all double points of $c_{2 n}$; the net $c_{n}$ defines upon it a $g_{2(n-1)}^{2}$. If $c_{2 n}$ has no other double points, the net defines an adjoint system. The canonical adjoint curves of order $2 n-3$ can be composed of $\phi_{n}$ and any fixed curve of order $n-3$. The usual form of the theorem of birational transformation is that any system of adjoint curves of order $2 n-3$ and having two degrees of freedom may be used for transforming curves, but for our net the double points furnish the minimum number of conditions for a curve of order $n$. Moreover, the $g_{2(n-1)}^{2}$ is of such a nature that one point determines $n-2$ others, analogous to the case of the hyperelliptic curves. If $n>3, p>2 n-2$, hence $c_{2 n}$ cannot be hyperelliptic.

2. When $n=3$, the $g_{4}^{2}$ is composed of two $g_{2}^{1}$, the groups being associated arbitrarily, hence it is hyperelliptic.

The necessary and sufficient condition that a sextic curve with seven double points be hyperelliptic is that every pencil of cubic curves through the seven double points and one simple point on the curve shall have the residual basis point on the curve also.

The curve can be reduced to a quintic with a triple point by means of adjoint quartics; it cannot be reduced to a quartic curve.

3 . If we now write $\rho x_{1}=c_{n} \chi_{n-3}, \rho x_{2}=c_{n}^{\prime} \chi_{n-3}, \rho x_{3}=c_{n}^{\prime \prime} \chi_{n-3}$ then (1) will go into a conic section, but it will be shown that (1) can never be rational. If we use (for a moment) the notation of Amodeo in his memoir on minimum adjoint curves (Rendiconti dei Lincei, series 5 , volume 2 (1893), pages $460-467)$ we have $m=2 n, p=n^{2}-2 n, \alpha=b-3, \rho=\frac{1}{2}(n-2)(n-3)$, wherein adjoint curves of $c_{m}$ of order $m-3-\alpha$ exist and will pass through the remaining $\rho$ double points if required to pass through all but $\rho$. As is shown by Amodeo, if these conditions are satisfied, then the intersection of $c_{2 n}$ with any $c_{n-3}$ (not adjoint), forming a $g_{2 n(n-3)}^{n / 2(n-3)}$, will not be a complete series, but will be contained in another having $\rho$ further degrees of freedom. Equation (1), page 466, is satisfied if $t=1$, hence all the formulas are satisfied when our $c_{2 n}$ has the maximum genus $n^{2}-2 n$.

4. Any two curves of the net will intersect in $n-1$ points lying on a straight line. The pencil of adjoint curves which has any fixed basis point on $c_{2 n}$ will have $n-2$ other fixed basis points upon it, and define a $g_{n-1}^{1}$. The $n-1$ points of each group of this series are collinear. Such a series can be defined by starting from any point of $c_{2 n}$ as basis point, but from 
the form of the equation it immediately follows that all the $g_{n-1}^{1}$ are identical. The groups of $g_{n-1}^{1}$ determine an $[n-2]$ involution on $c_{2 n}$. If $p$ be the genus of $c_{2 n}$, the number of coincidences is $2(n+p-2)$.*

If $c_{2 n}$ has a double point apart from the $n^{2}-n+1$ basis points, it will count for two coincidences, hence if $c$ be the number of simple coincidences we have

or

$$
2(n+p-2)=2\left(n^{2}-2 n-p\right)+c
$$

$$
4 p=2 n(n-3)+c+4 .
$$

Since $c$ cannot be negative, the minimum value of $p$ is that of the general $c_{n}$, thus

$$
\frac{1}{2}(n-1)(n-2) \leqq p \leqq n(n-2) .
$$

If singular $G_{n-1}$ exist in $g_{n-1}^{1}$, double points may arise which do not involve two coincidences, but they can only occur for particular relations among the coefficients of $c_{2 n}$, which we exclude.

Since $p>0$, we can now state the following results:

(a) The complete series $g_{2(n-1)}^{2}$ cannot be used for birational transformation.

(b) The envelope of the straight lines on which lie the groups of $g_{n-1}^{1}$ is a conic. $\dagger$

(c) The normal or canonical form of $c_{2 n}$ is a conic, taken $(n-1)$ fold.

The analogy between these curves and the hyperelliptic curves thus becomes apparent.

5. When $n=4$, it was seen that the straight lines of the plane cut from $c_{8}$ a partial series $g_{8}^{2}$ contained in the complete series $g_{8}^{3}$, hence $c_{8}$ is the projection of a space curve of the same order. Curiously, the same thing is true for every value of $n$. Consider the space curve on the quadric surface, cutting the generators of one system in $n+1$ points, and those of the other in $n-1$ points. The space curve $R_{2 n}$ has therefore $h=n^{2}-n+1$

* From the Cayley-Brill principle of correspondence, or from Zeuthen's formula. See Bertini : "La geometria delle serie lineari sopra una curva piana secondo il metodo algebrica," Annali di Mat. (2), vol. 22 (1894), pp. 1-40.

† Amodeo, "Curve $\kappa$-gonali," Ann. di Mat. (2), vol. 21 (1893), pp. 221336. See formula, bottom page 231. $m=2 n, \kappa=n-1, t=1$, hence $y$, the class of the envelope, is 2 . 
apparent double points. If now we project into $c_{2 n}$ from a center not on the quadric, the $n^{2}-n+1$ double points will be distinct. We thus have the theorem: "If through $h-\frac{1}{2} i(i+1)$ of the $h$ double points of the projections curve $c_{2 n}$ a curve of order $2 n-i-3$ can be passed $(2 n-i-3 \geqq n-1)$, then this curve will pass through the remaining $\frac{1}{2} i(i+1)$ double points also."*

Through $\frac{1}{2} n(n+3)-2$ arbitrary points can be passed $\infty^{2}$ $c_{n}$. If $i=n-3$, we see from the above theorem that all such curves $c_{n}$ through $\frac{1}{2} n(n+3)-2$ of the double points will pass through the others also, hence a net of adjoint curves of order $n$ exists for our projection curve $c_{2 n}$. The adjoint curves define upon $c_{2 n}$ a $g_{2(n-1)}^{2}$. In case $n=4$, the $g_{6}^{2 *}$ is the residual of $g_{8}^{3}$ by the Riemann-Roch theorem, but in no case can this net be used to transform the curve $c_{2 n}$ birationally into another of order $2(n-1)$. If we project $R_{2 n}$ from a point of the quadric on which it lies, $c_{2 n}$ will have an $(n-1)$ fold point $P_{n-1}$ and a $P_{n+1}$, and in general no other singularities. The $g_{n-1}^{1}$ defined by one system of generators is thus defined by one pencil of lines, and the $g_{n+1}^{1}$ by another. By projecting $R_{2 n}$ from a point upon the curve, or by ordinary inversion of $c_{2 n}$ we obtain a $c_{2 n-1}$ with a $P_{n}$ and a $P_{n-2}$, and no other singularities. By inversion, an infinite number of projectively distinct $c_{2 n-1}$ can be obtained, but they will all have multiple points of the same order. In no case can this curve be reduced to a curve of lower order by any birational transformation. $\dagger$

When $n=4, c_{8}$ has a $g_{3}^{1}$ which is invariant, but when $c_{m}$ has a $g_{3}^{1}, p \leqq 2 m-5$; since $c_{8}$ is of genus 8 , it follows that $m \stackrel{m}{\geqq} 7$.

Probably similar deductions can be made for larger values of $n$ from the memoirs of Amodeo and of Bertini. Any adjoint $\phi_{n}$ containing a point $P$ must also contain the whole group $G_{n-1}$ to which $P$ belongs (Bertini, l. c., page 29), hence if two different $\phi_{n}$ pass through $P$, the $n-1$ points of intersection will be the $G_{n-1}$ to which $P$ belongs. These points always lie on a straight line (Bertini, page $30, b$ ). This shows that our projection curve $c_{2 n}$ can be generated projectively by the method of $\S 1$, and the

* H. Valentiner, "Zur Theorie der Raumkurven," Acta mat., vol. 2 (1883), pp. 136-230. See p. 177. Also M. Noether, "Zur Grundlegung der Theorie der algebraischen Raumkurven," Berliner Abhandlungen (1883), pp. 1-120. See p. 24.

† V. Snyder. "On birational transformation of curves of high genus," Amer. Jour. Nath., vol. 30 (1908), pp 11-20.

$\ddagger$ K. Bobek : “Ueber Dreischaarkurven," Wiener Berichte, vol. 98 (1889), pp. 141-172. 
two systems are thus coextensive. Since on $R_{2 n}$ the $g_{n-1}^{1}$ is defined by the generators of one system of the hyperboloid, the envelope of the lines containing the $G_{n-1}$ is the section of the tangent cone to the hyperboloid from the center of projection.

6 . If $\phi_{n}, \phi_{n}^{\prime}, \phi_{n}^{\prime \prime}$ be three cones of order $n$ containing the $n$ bisecants from $(0,0,0,1)$, then, since the $n-1$ remaining edges of intersection with the cone $R_{2 n}$ from the same point lie in a plane, the equation of the defining monoid may be written

$$
w=\frac{x \phi_{n}^{\prime}}{\phi_{n}}=\frac{x \phi_{n}^{\prime \prime}}{\phi_{n}^{\prime}},
$$

from which the equation (1) results. Incidentally, these equations furnish a means for reducing $c_{2 n}$ to $c_{2 n-1}$, namely, the $\infty^{2}$ plane sections of the monoid from a point on $R_{2 n}$, lying on one of the $n-1$ simple edges.

7. If $R_{2 n}$ has also actual double points or cusps, $\phi_{n}$ will not in general pass through them, hence in the plane curves $c_{2 n}$ we can distinguish between projection of actual double points and apparent double points. Actual double points will not always absorb two coincidences in the $[n-2]$ involutions, but when $p<\frac{1}{2}(n-1)(n-2)$, the projection curve can not be written in the form (1).

For other curves on the hyperboloid, the maximum number of basis lines of a net formed by bisecants will not be reached; but when no actual double points occur we may say that the projection curve $c_{n}$ with $p>\frac{1}{6}(n-1)(n-2)$ cannot be birationally transformed into any curve of order less than $n-1$.

CORNell UNIVERSITY, August, 1907.

\section{NOTE ON CERTAIN INVERSE PROBLEMS IN THE SIMPLEX THEORY OF NUMBERS.}

BY PROFESSOR R. D. CARMICHAEL.

(Read before the American Mathematical Society, September 5, 1907.)

Legendre* has considered the problem of finding the highest power of a prime $p$ contained in $m !=1 \cdot 2 \cdot 3 \cdots m$. Let $m$ be written in the form

$$
m=a_{0} p^{\alpha}+a_{1} p^{\beta}+a_{2} p^{\gamma}+\cdots,
$$

* A. M. Legendre, Théorie des nombres, 3d ed., I., p. 10. 